\title{
Discussion on the Methods of Improving Computer Teaching
}

\author{
Qinghua Xu
}

\begin{abstract}
Jiangxi Vocational Technical College of Industry \& Trade, Jiangxi, Nanchang, 330038
739960103@qq.com
\end{abstract}

\begin{abstract}
Want to improve the students' computer learning efficiency, first of all is the key to stimulate students interest in computer learning, let the students enthusiasm for learning computer, so that they can give full play to the ability of autonomic learning to active learning, if teachers computer classroom teaching as their own centralized, it is difficult to make students produce strong interest in computer, so under the new education idea, the teacher should learn to the initiative back to student, should give priority to in order to guide students to learn, in the process of students' learning support. In order to improve the quality and efficiency of computer teaching, it is also necessary to discuss the methods of computer teaching. Therefore, this paper mainly focuses on the methods that can effectively improve computer teaching.
\end{abstract}

Keywords: computer teaching, improvement, discussion

\section{基于提高计算机教学的方法探讨}

\author{
徐庆华
}

\author{
江西工业贸易职业技术学院 江西 南昌 330038 \\ 739960103@qq.com
}

\section{摘要}

想要提高学生的计算机课程学习效率, 首先比较关键的就要激发学生对计算机学习的兴趣, 让学生对计算机学 习充满热情, 从而能够充分发挥自主学习能力进行主动学习, 如果教师只是将计算机教学课堂当做自己的一言 堂, 这就很难使学生对计算机产生浓厚的兴趣, 因此在新的教育理念下, 教师应该将学习的主动权交还给学生, 要以引导学生学习为主, 在学生学习的过程中起到支持作用。而为了提升计算机教学的质量和效率, 对计算机 教学的方法的探讨也是十分必要的, 因此, 本文也主要围绕能够有效提升计算机教学的方法进行研究和探讨。 关键词: 计算机教学; 提高; 探讨

\section{1 计算机教学现状}

科学技术的发展推动了信息时代的到来, 而在信 息时代背景下, 计算机技术在人们的生活中已经担任 了不可或缺的重要角色。无论是在人们的生活中学习 中还是工作中, 甚至是在社会的发展与进步中, 计算 机技术都提供了非常大的帮助。因此计算机技术在我 国的应用范围也是非常广泛的, 无论是教师还是学 生, 都有必要具备一定的计算机技术应用能力, 而对 于计算机专业教师而言, 则应该不断更新自己的知识 体系, 确保自己所获取到的都是最先进的计算机知
识, 这样才能够更好的带领学生, 探索计算机领域的 奥妙。而仅仅是对于非计算机专业的学生而言, 计算 机能力也是必不可少的, 具备计算机应用能力的学生 在就业市场上也能更加有竞争力。但是当前计算机课 堂教学已然陷入困境, 教师难以调动起学生的学习兴 趣, 学生的学习情况也不容乐观。而会产生这种情况, 并不只是因为我国计算机教育教学发展缓慢, 更因。 我国计算机教育教学的方式与当下的市场需求不符。 学生学习计算机知识是为了更好的应用计算机知识, 也是为了在走上工作岗位之后能够更好的运用计算 机知识解决现实问题。而高校却模糊了重点, 更加注 
重对学生的计算机理论知识的教学, 使得学生所具备 的能力与市场所需要的能力并不匹配。而除此之外, 当前我国教育教学领域可以运用的教学手段非常丰 富, 但是在计算机教育教学中使用的却还是传统的教 育教学手段, 在传统的教育教育手段面前, 计算机知 识的趣味性没能得到完全的展现, 反而更容易使学生 觉得计算机知识枯燥无味, 并心生厌倦。

为了能够更好的调动学生的学习积极性, 也为了 能够使计算机教育教学质量和效率能够达到应有的 水平, 教师应该学习更加先进的计算机知识, 也应该 与学生之间构建良好的师生关系, 如果教师能够成为 学生的榜样, 则能够将这种师生关系的有益性发挥到 最大。当教师成为学生的榜样, 学生会不由自主的跟 随教师, 模仿教师, 并尽可能的提升自己的学习成绩, 提高自己的应用水平, 而这样一来, 学生学习的动力 就属于自发性动力, 不需要教师和家长的督促, 因此 在这种情况下, 学生的学习成果也是最可观的。而建 立良好的师生关系的前提是教师应该展现自己在专 业上的专业魅力, 首先要让学生认识到教师的专业 性, 并认同教师的专业能力, 这样学生就很容易对教 师产生崇拜之情, 而其次, 教师还应该多多关怀学生, 多和学生沟通交流, 让学生感受到教师的温暖, 除此 之外, 教师还应该多多鼓励学生, 在学生遇到挫折, 又或是面对难关时, 教师都应该鼓励学生不要放弃, 勇往直前, 只有当学生解决了一些问题之后, 学生才 能找到学习成就感, 并继而更加热爱学习, 而在学生 解决问题的过程中, 教师不应该插手，但可以适当的 给予学生一些指导。

\section{2 提高计算机教学的有效方法}

\section{表 1 提高计算机教学的有效方法}

\begin{tabular}{|c|c|c|}
\hline \multicolumn{3}{|c|}{ 提高计算机教学的有效方法 } \\
\hline $\begin{array}{c}\text { 采用分组教学, } \\
\text { 增强学生的团 } \\
\text { 队协作精神 }\end{array}$ & $\begin{array}{c}\text { 采用互动式教学, } \\
\text { 提高学生的学习 } \\
\text { 热情 }\end{array}$ & $\begin{array}{l}\text { 适时活跃课堂 } \\
\text { 气氛, 集中学 } \\
\text { 生的注意力 }\end{array}$ \\
\hline $\begin{array}{c}\text { 在学生上机实 } \\
\text { 践过程中, 有意 } \\
\text { 识的把学生分 } \\
\text { 成几个小组可 } \\
\text { 以促进学生的 } \\
\text { 共同提高。大家 } \\
\text { 都想为本小组 } \\
\text { 争光, 希望能成 } \\
\text { 为最好的小组, } \\
\text { 所以他们的注 } \\
\text { 意力高度集中, } \\
\text { 上机时间就能 } \\
\text { 得到有效的利 } \\
\text { 用。 }\end{array}$ & $\begin{array}{c}\text { 为了能够与学生 } \\
\text { 互动, 老师应该首 } \\
\text { 先充满激情, 用抑 } \\
\text { 扬顿挫的音调来 } \\
\text { 给学生上课, 并适 } \\
\text { 时的走到学生的 } \\
\text { 中间, 让学生感受 } \\
\text { 到你的激情, 让学 } \\
\text { 生知道你在注意 } \\
\text { 着并且很重视他 } \\
\text { 们对所讲知识的 } \\
\text { 反应。 }\end{array}$ & $\begin{array}{l}\text { 调查结果显示, } \\
\text { 近六成的学生 } \\
\text { 上课集中精力 } \\
\text { 的时间达不到 } \\
30 \text { 分钟。长时 } \\
\text { 间的集中注意 } \\
\text { 力, 会使学生处 } \\
\text { 于一种疲劳的 } \\
\text { 状态, 影响听课 } \\
\text { 效果, 这就需要 } \\
\text { 老师适时的来 } \\
\text { 调节课堂氛围。 }\end{array}$ \\
\hline
\end{tabular}

\section{1 采用分组教学, 增强学生的团队协作精神}

学生与学生之间的学习水平和学习基础是存在差 距的, 因此教师也应该按照学生的不同的学习水平和学 习基础将学生划分为几个不同的层次。对学生进行针对 性教学。但是在大班教学模式下, 分层次教学法。往往 很难取得其有效性。所以教师还可以采用分组教学的方 式, 将学生分为若干个小组, 让学生在完成学习任务的 过程中得到提升。而这样的方式, 无论是学习水平高或 是学习水平低的学生, 在团队中都能够起到一定的作 用, 也都能够学习到更多的知识。并且, 小组学习方式 能够激发学生的荣誉感, 让学生更加投入到学习, 。而 在这个过程中, 教师所需要做的只是发布学习任务, 监 督学生的完成情况。以及指导学生一些难度较大的问 题。

\section{2 采用互动式教学, 提高学生的学习热情}

计算机课程是一门实践性课程, 而理论性教学固然 重要, 实践教学也必不可少, 而想要让学生更加深入的 掌握教学内容, 教师就必须做到及时掌握学生的学习进 度，因为不同学生的吸收速度不同，教师应该根据学生 的吸收知识的速度来判断学生学习的效率, 并使不同阶 段的学生都能够掌握教学内容, 因此这也要求教师要不 断地对教学方案进行调整, 而为了使学生在课堂上能够 集中注意力, 教师还应该丰富自己的教学手段, 并且使 自己的教学方式更有感染力, 能够吸引学生的目光。

\section{3 适时活跃课堂气氛, 集中学生的注意力}

大部分的学生在课堂上都很难集中注意力, 一方面 是学生的天性使然, 另一方面是因为教学内容不能够引 起学生的兴趣。而一节课的时间往往是学生能够集中注 意力的时间的好几倍。因此学生总是容易在教学的中段 走神或是瞌睡。而每当出现这种情况。老师作为教学者 不应该气馁, 而是应该想办法吸引学生的注意力。例如 采用提问的方式, 来引导学生思考, 学生在思考的过程 中就不容易走神, 或是犯困。为了将提问的有效性发挥 到最大, 教师应该尽可能的选择学生已学习过的, 又不 是学生有所了解的内容进行提问。如果问题太难, 则更 容易使学生感到抗拒, 而如果问题太简单, 就起不到吸 引学生注意力的作用, 所以在选择问题方面, 教师也应 该花费更多的精力。

\section{4 对多媒体信息技术进行充分利用}

在计算机教学的过程中要注重对当代多媒体信息 技术的应用, 通过对传统教学方式进行一定的变革, 将 多媒体技术中的文字、图像、音视频动画等等多方向的 多媒体技术应用到一起，提高计算机课堂教学的有效 性。要多加利用线上教学工具，加强教师和学生之间的 沟通和交流, 通过将线上学习资料进行充分利用, 提升 学生的学习积极性, 更好的理解课堂教学知识, 接近教 师的教学目标。 


\section{5 采用项目式教学以促进学生的学习主观 能动性}

项目制学习是通过项目分配的方式, 让学生独立自 主完成整个学习任务, 在这个过程中能够充分发挥学生 的主观能动性, 激发学生的学习热情, 从而进行自主探 索和学习。将学生以往的被动学习状态转变为主动学 习, 让学生在项目实践的过程中自主探索问题的答案, 在学习过程中带着问题进行学习, 教师在这个过程中起 到的作用就是辅助作用, 主要是让学生进行自发性学习 和解决问题, 以达到提升学习效率的作用。

\section{6 教师要发自内心关心学生, 和学生建立起 好的情感联结}

师生关系对学生的学习效率起到至关重要的作用, 师生之间的关系如果严肃呆板, 课堂教学氛围不轻松, 就无法激发学生的学习积极性, 学生对教师并不感兴 趣, 学习效率就得不到保障, 师生之间的关系如果足够 融洽, 学生就会想要接触和亲近教师, 同样也会对老师 所传授的知识感兴趣, 这样学生的学习积极性就会大大 提升, 并且也会愿意向老师提出自己的疑惑。教师想要 与学生建立起良好的师生关系就需要发自内心的喜欢 教师这个行业, 真正关心学生, 多给学生以鼓励, 将学 生当做朋友来相处。师生之间只有建立起和谐的关系, 学生的学习积极性就会提升, 学习效率也会有所提高。

\section{7 改变教学理念, 坚持以学生为中心进行教 学活动的开展}

计算机教学的有效开展需要教师有先进的教学观 念, 其中最基本的就是要以学生为中心进行计算机课程 教学的开展, 要想做到以学生为中心首先就需要做到根 据学生所学习的专业进行针对性教学, 同时要采取一些 当代先进的教学方法和教学工具, 将传统教学课堂中的 一些过时的教学内容和教学模式进行删选, 这样就可以 教给学生贴合当代社会发展的专业的计算机知识, 这样 在学生出学校校门后也可以在工作中直接应用所学知 识, 能够找到理想的工作。所以提高计算机教学方法是 十分必要的, 可以教给学生理论知识的同时也可以让学 生具备很好的专业实践能力。

\section{3 结论}

教师的教学内容和教学目标应该按照社会的人才 需求来制定, 并为了提高计算机专业教学的有效性, 有 必要激发学生的学习兴趣, 培养学生良好的学习习惯, 虽然目前我国计算机专业教学中还存在着很多的问题, 这些问题在某种程度上阻碍着学生的发展, 但是相信通 过不断的探索, 能够找到提升计算机专业教学有效性的 最佳途径。

\section{REFERENCES}

[1] Wu Meng. Research on the Reform of Computer-Aided Design Course Teaching Method under the Background of New Era Education [J].
Science, Technology and Economics Review, 20,28(32): 195+194.

[2] Lu Hui, Li Hua, Ma Xuebin. Design and Application of Micro Flipping Teaching Method for Computer Network Principle Problems Based on Innovative Talent Training [J]. Computer Education, 2020(07): 74-78.

[3] Xu Feng. Reflections on the Innovative Methods of Computer Teaching in Secondary Vocational Schools under the Situation of Employment [J]. Popular Science Fairy Tales,2020(28):30.

[4] Han Jing. Research on the Application of Task-driven Teaching Method in the Teaching of Information Technology Majors in Secondary Vocational Schools [D]. Shanxi University,2020.

[5] Hou Liyan. Research on the Integration and Application of Multiple Teaching Methods in the Teaching of "Computer Fundamentals" in the Information Age $[\mathrm{J}]$. Wireless Internet Technology,2020,17(10):95-96.

[6] Yang Aimin, Cen Zhongdi, Wang Renfang. Research on the construction of computer experimental teaching demonstration center under the background of new engineering $[\mathrm{J}]$. Computer Education,2020(05):64-67.

[7] Song Yubo. The Method of Cultivating Students' Innovative Ability in Computer Teaching in Secondary Professional Schools [J]. Digital Communications World,2020(05):283.

[8] Ouyang Yuanxin, Liu Yu, Wu Xiujuan, Zhang Bolin, Cao Qinghua. Research on the Teaching Method of "College Computer Fundamentals" in Social Sciences and Situational Case Design [J]. Information Technology Education in China, 2020(09): 104-106.

[9] Wu Xihong. Improving Teaching Methods and Improving Classroom Teaching Quality -- Taking Computer Disciplines as Example [J]. Computer Products and Circulation,2020(02):165-166.

[10] Liu Yagang, Liu Shuhui. Computer course teaching reform and research based on the cultivation of computational thinking ability $[\mathrm{J}]$. Electronic Testing,2020(02):116-118.

[11] Chen Jianjun, Wang Qi, Hu Chunlong, Zuo Xin, Jiang Dengbiao, Liu Yuan. Research and Practice on Teaching Methods of Computer Programming Course for Overseas Undergraduates [J]. Forum on Education and Teaching,2020(01):202-203.

[12] Zheng Yi. Application of Task Driven Teaching Method in "Computer Interlocking Experiment" 
Course [J]. Research in Contemporary Educational Practice and Instruction,2019(24):144-145.

[13] Liu Yu. How to Cultivate Students' Creative Thinking Ability in Computer Teaching with Flexible Teaching Methods [J]. Professional, 2019 (35): 68-69.

[14] Wen Liying, Fang Yu, He Yuwan. Research on Teaching Method of Computer Basic Course for
International Students Based on SPOC+ Flipped Classroom $\quad[\mathrm{J}]$. Computer Education,2019(12):93-96.

[15] Pei Ying. Practice of Strengthening the Teaching of Computer Basic Courses in Colleges and Universities -- Research on Big Data in Computer Basic Teaching Methods [J]. Journal of Computer Science and Technology,2019,15(31):166-167. 